\title{
Observations of mutual phenomena of Galilean's satellites at Catania
}

\author{
Daniele Fulvio and Carlo Blanco
}

Physics and Astronomy Department, Catania University, via S. Sofia 64, 95123 Catania, Italy

INAF - Catania Astrophysical Observatory, via S. Sofia 78, 95123 Catania, Italy

email: dfu@oact.inaf.it

c.blanco@oact.inaf.it

\begin{abstract}
The mutual phenomena between Jupiter and Saturn's satellites occur every half orbital period of these planets, when the Earth and the Sun cross their equatorial plane. At Physics and Astronomy Department of Catania University the events between Jupiter's satellites have been observed during the 1973, 1979, 1985/86, 1991, 1997 and 2009 campaigns and the ones between Saturn's satellites during the 1980/81 and 1995 campaigns. An overview of the main results obtained since 1973 is presented.
\end{abstract}

Keywords. techniques: photometric, telescopes, planets and satellites: Jupiter, planets and satellites: galilean satellites

\section{Introduction}

Prior to the Voyager missions the possibility to define the physical parameters and the surface morphology of the moons of the greatest Solar System planets was entrusted to the few photographs or to the drawings by astronomers. The lightcurves of the mutual phenomena give the objective data to better define the surface characteristics. As an example, the comparison between the albedo of the eclipsed areas and the drop in magnitude in different colors, the duration and the time of the minimum of light inferred, during the 1973 campaign, the existence of bright polar caps on Io, Europa and Ganymede, suggested by the drawings of many authors.

Since the Voyager missions have provided accurate physical parameters for all the satellites and described surface features and albedo variations, the observations of mutual events were best used for astrometric purposes. As an example, during the 1979 campaign, the relative separations in right ascension, $\Delta \alpha \cos \delta$, and declination, $\Delta \delta$, of a satellite pair at midevent were estimated.

The improvement of the integration time up to measures of the order of $0.1 \mathrm{sec}$, can supply relative positions with relevant residuals near $100 \mathrm{Km}$ at Jupiter's mean distance, with the possibility, due to the great number of observed events, to evidence tidal effects on Io's orbit.

After the 1980 campaign, the observing conditions were completely changed by the incoming of the CCD detectors. Their spatial resolution of the order of arcsec per pixel, by the analysis of the lightcurve behavior during the event, also with small telescopes, makes it possible to obtain morphological details of the order of few kilometers. The use of several infrared wavelengths during Io's occultations by the other satellites, allows the detection of emitting hot spots associated to an intense volcanic activity on the first Galilean satellite of Jupiter. 


\section{Previous observational campaigns}

At Physics and Astronomy Department of Catania University the events between Jupiter's satellites have been observed during the 1973, 1979, 1985/86, 1991, 1997 and 2009 campaigns. All the observations were carried out at M. G. Fracastoro Station of INAF - Catania Astrophysical Observatory.

During the 1973 campaign, the 61/41-cm Schmidt telescope equipped with a synchronous three-channel photometer able to obtain contemporaneous observations in the $U B V$ bands (Blanco \& Catalano 1974) was used. Two mutual occultation and one mutual eclipse were observed (see Table 1). The observed depth of all events is shallower than the predicted one by about $0^{m} .2$, apart from the JII occultation on October $22^{\text {nd }}$ 1973, when the observed depth is much deeper than the predicted one. However, the observations of this event were made in the $B$ band while the predictions refer to the $V$ magnitude. The observed times of occurrence for all events are in close agreement with the predictions by Aksnes 1974.

Since the 1979 campaign, the 91-cm Cassegrain telescope was used and since the 1985 campaign it was equipped with photon counting photoelectric photometer.

Table 1. Relevant data on the events observed during the 1973 campaign (Blanco \& Catalano 1974). Predicted values of the light minimum (UT) and the light loss were taken by Aksnes (1974).

\begin{tabular}{cccccc}
\hline Date & Event & $\begin{array}{c}\text { Predicted } \\
\text { minimum }\end{array}$ & $\begin{array}{c}\text { Observed } \\
\text { minimum }\end{array}$ & $\begin{array}{c}\text { Predicted } \\
\text { Light loss }\end{array}$ & $\begin{array}{c}\text { Observed } \\
\text { Light loss }\end{array}$ \\
\hline OCT 22 & 3O2P & $17^{h} 43^{m}$ & $17^{h} 43^{m} 45^{s} \pm 0^{s} .3$ & $0^{m} .01(\Delta \mathrm{V})$ & $0^{m} .08 \pm 0^{m} .04(\Delta \mathrm{B})$ \\
NOV 15 & $4 \mathrm{E} 3 \mathrm{~T}$ & $18^{h} 10^{m}$ & $18^{h} 09^{m} 45^{s}$ & $0^{m} .80(\Delta \mathrm{V})$ & $0^{m} .615 \pm 0^{m} .03(\Delta \mathrm{V})$ \\
& & & & & $0^{m} .625 \pm 0^{m} .03(\Delta \mathrm{B})$ \\
NOV 22 & 2E1A & $16^{h} 55^{m}$ & $16^{h} 53^{m} 10^{s}$ & $0^{m} .73(\Delta \mathrm{V})$ & $0^{m} .660 \pm 0^{m} .03(\Delta \mathrm{U})$ \\
& & & & & $0^{m} .530 \pm 0^{m} .03(\Delta \mathrm{V})$ \\
& & & & & $0^{m} .450 \pm 0^{m} .03(\Delta \mathrm{B})$ \\
& & & &
\end{tabular}

Table 2. Relevant observed data on the 1979 mutual events of Jupiter satellites (Blanco 1996).

\begin{tabular}{cccccc}
\hline Date & Event & $\Delta \alpha \cos \delta$ & $\Delta \delta$ & $\begin{array}{c}\text { Duration } \\
(\mathbf{s e c})\end{array}$ & $\begin{array}{c}\text { Observed } \\
\text { Light loss }\end{array}$ \\
\hline OCT 01 & 1E2P & +0.072 & +0.182 & $445 \pm 0^{s} .3$ & $1^{m} .80 \pm 0^{m} .05(\Delta \mathrm{V})$ \\
& & & & $1^{m} .83 \pm 0^{m} .05(\Delta \mathrm{B})$ \\
NOV 02 & 1E2P & -0.160 & -0.390 & 260 & $1^{m} .65 \pm 0^{m} .05(\Delta \mathrm{U})$ \\
& & & & $0^{m} .80 \pm 0^{m} .03(\Delta \mathrm{V})$ \\
NOV 02 & 3E2P & +0.094 & +0.321 & 140 & $0^{m} .80 \pm 0^{m} .03(\Delta \mathrm{B})$ \\
& & & & $0^{m} .75 \pm 0^{m} .03(\Delta \mathrm{U})$ \\
NOV 09 & 1E2P & -0.262 & -0.635 & 235 & $1^{m} .10 \pm 0^{m} .03(\Delta \mathrm{V})$ \\
& & & & $1^{m} .12 \pm 0^{m} .03(\Delta \mathrm{B})$ \\
& & & & $0^{m} .20 \pm 0^{m} .03(\Delta \mathrm{V})$ \\
& & & & $0^{m} .17 \pm 0^{m} .03(\Delta \mathrm{B})$ \\
& & & &
\end{tabular}

Relevant observed data on the 1979 mutual events of Jupiters satellites are reported in Table 2 (Blanco 1996). Due to Catania geographic coordinates, the 1979 apparition was a poor one, requiring most observations to be made through large air masses, thus 
resulting in uncertain sky corrections and approximate lightcurves. The observations of mutual events of this campaign were best used for astrometric purposes.

A total of 37 mutual events were observed in 1985 and 22 of these were of such good quality to improve the computations of satellites' orbits. These events are reported in Table 3 (Blanco 1988; Franklin et al. 1991). Predicted values of the light minimum (UT) here shown were computed by Arlot (1984) by using the ephemerides given by Arlot (1982). The predicted light losses were by Aksnes \& Franklin (1984). The observed light losses and values of the light minimum do not show relevant regular deviations from the predicted ones.

During the 1991 campaign we observed 29 mutual events. In Table 4 we report relevant data for some of them (Arlot et al. 1997).

As part of the PHEMU97 International Campaign, 28 lightcurves were obtained at M. G. Fracastoro Station. The mutual events that present little noise and allowed us to compare themself with predictions are 10 and they are reported in Table 5 (Blanco 1999; Blanco et al. 2001; Arlot et al., 2006). A global analysis of these data shows that the times of occurrence of the observed minima normally come early compared to the predicted ones. Moreover, the observed light losses in seven cases are greater than the predicted ones while they are comparable in the remaining three cases.

Table 3. Mutual events observed in 1985 (Blanco 1988; Franklin et al. 1991). Predicted values of the light minimum (UT) here shown were computed by Arlot (1984) by using the ephemerides given by Arlot (1982). The predicted light losses were by Aksnes \& Franklin (1984).

\begin{tabular}{|c|c|c|c|c|c|}
\hline Date & Event & $\begin{array}{l}\text { Predicted } \\
\text { minimum }\end{array}$ & $\begin{array}{l}\text { Observed } \\
\text { minimum }\end{array}$ & $\begin{array}{c}\text { Predicted } \\
\text { Light loss }(\Delta \mathrm{V})\end{array}$ & $\begin{array}{c}\text { Observed } \\
\text { Light loss }(\Delta \mathrm{V})\end{array}$ \\
\hline JUN 17 & $3 \mathrm{O} 4 \mathrm{P}$ & $01^{h} 44^{m} .91$ & $01^{h} 45^{m} .24 \pm 0^{m} .005$ & $0^{m} .09$ & $0^{m} .06 \pm 0^{m} .03$ \\
\hline JUL 08 & $3 \mathrm{O} 2 \mathrm{P}$ & $22^{h} 17^{m} .21$ & $22^{h} 18^{m} .20$ & $0^{m} .31$ & $0^{m} .36 \pm 0^{m} .01$ \\
\hline JUL 12 & $4 \mathrm{O} 3 \mathrm{P}$ & $00^{h} 39^{m} .86$ & $00^{h} 39^{m} .90$ & $0^{m} .45$ & $0^{m} .32 \pm 0^{m} .01$ \\
\hline JUL 16 & $3 \mathrm{O} 2 \mathrm{P}$ & $01^{h} 14^{m} .31$ & $01^{h} 14^{m} .19$ & $0^{m} .27$ & $0^{m} .26 \pm 0^{m} .08$ \\
\hline AUG 05 & $3 \mathrm{O} 4 \mathrm{P}$ & $23^{h} 26^{m} .96$ & $23^{h} 28^{m} .85$ & $0^{m} .11$ & $0^{m} .04 \pm 0^{m} .01$ \\
\hline AUG 27 & $3 \mathrm{O} 2 \mathrm{P}$ & $19^{h} 36^{m} .95$ & $19^{h} 36^{m} .60$ & $0^{m} .18$ & $0^{m} .25 \pm 0^{m} .01$ \\
\hline AUG 29 & $4 \mathrm{O} 1 \mathrm{P}$ & $20^{h} 18^{m} .57$ & & $0^{m} .02$ & $0^{m} .03 \pm 0^{m} .01$ \\
\hline AUG 30 & $1 \mathrm{E} 2 \mathrm{P}$ & $20^{h} 03^{m} .90$ & $20^{h} 02^{m} .11$ & $0^{m} .40$ & $0^{m} .20 \pm 0^{m} .01$ \\
\hline SEP 03 & $3 \mathrm{O} 2 \mathrm{P}$ & $22^{h} 03^{m} .47$ & $22^{h} 03^{m} .72$ & $0^{m} .15$ & $0^{m} .17 \pm 0^{m} .01$ \\
\hline SEP 04 & $3 \mathrm{E} 2 \mathrm{~T}$ & $21^{h} 46^{m} .91$ & $21^{h} 54^{m} .36$ & $1^{m} .00$ & $0^{m} .25 \pm 0^{m} .01$ \\
\hline SEP 06 & $1 \mathrm{O} 2 \mathrm{P}$ & $21^{h} 49^{m} .45$ & $21^{h} 51^{m} .88$ & $0^{m} .04$ & $0^{m} .03 \pm 0^{m} .01$ \\
\hline SEP 07 & $1 \mathrm{E} 2 \mathrm{P}$ & $00^{h} 29^{m} .73$ & $00^{h} 29^{m} .67$ & $0^{m} .59$ & $0^{m} .25 \pm 0^{m} .02$ \\
\hline SEP 07 & $103 \mathrm{P}$ & $20^{h} 33^{m} .75$ & $20^{h} 34^{m} .07$ & $0^{m} .05$ & $0^{m} .03 \pm 0^{m} .01$ \\
\hline SEP 07 & $1 \mathrm{E} 3 \mathrm{P}$ & $23^{h} 22^{m} .31$ & $23^{h} 22^{m} .02$ & $0^{m} .31$ & $0^{m} .26 \pm 0^{m} .01$ \\
\hline OCT 01 & $4 \mathrm{E} 2 \mathrm{~A}$ & $19^{h} 06^{m} .66$ & $19^{h} 07^{m} \cdot 20$ & $0^{m} .68$ & $0^{m} .34 \pm 0^{m} .01$ \\
\hline OCT 01 & $1 \mathrm{E} 2 \mathrm{~A}$ & $21^{h} 52^{m} .16$ & $21^{h} 51^{m} .08$ & $0^{m} .94$ & $0^{m} .92 \pm 0^{m} .02$ \\
\hline OCT 02 & $4 \mathrm{E} 1 \mathrm{~A}$ & $19^{h} 33^{m} .11$ & $19^{h} 35^{m} .00$ & $0^{m} .81$ & $0^{m} .85 \pm 0^{m} .01$ \\
\hline OCT 31 & $3 \mathrm{E} 1 \mathrm{P}$ & $19^{h} 17^{m} .06$ & $19^{h} 16^{m} .29$ & $0^{m} .47$ & $0^{m} .46 \pm 0^{m} .01$ \\
\hline NOV 07 & $2 \mathrm{E} 1 \mathrm{~A}$ & $16^{h} 52^{m} .53$ & $16^{h} 52^{m} .09$ & $0^{m} .55$ & $0^{m} .65 \pm 0^{m} .01$ \\
\hline NOV 14 & $2 \mathrm{E} 1 \mathrm{~A}$ & $19^{h} 06^{m} .56$ & $19^{h} 06^{m} .36$ & $0^{m} .58$ & $0^{m} .68 \pm 0^{m} .02$ \\
\hline DEC 04 & $1 \mathrm{O} 2 \mathrm{P}$ & $17^{h} 18^{m} .31$ & $17^{h} 18^{m} .23$ & $0^{m} .10$ & $0^{m} .08 \pm 0^{m} .01$ \\
\hline DEC 14 & $3 \mathrm{E} 2 \mathrm{P}$ & $17^{h} 34^{m} .66$ & & $0^{m} .98$ & $0^{m} .77 \pm 0^{m} .03$ \\
\hline
\end{tabular}


Table 4. Relevant data of some mutual events observed during the 1991 campaign (Arlot et al. 1997). Predicted values of the light minimum (UT) and the light loss were by Arlot (1997).

\begin{tabular}{cccccc}
\hline Date & Event & $\begin{array}{c}\text { Predicted } \\
\text { minimum }\end{array}$ & $\begin{array}{c}\text { Observed } \\
\text { minimum }\end{array}$ & $\begin{array}{c}\text { Predicted } \\
\text { Light loss }(\Delta \mathbf{V})\end{array}$ & $\begin{array}{c}\text { Observed } \\
\text { Light loss }(\Delta \mathbf{V})\end{array}$ \\
\hline JAN 02 & 2O3A & $01^{h} 40^{m} 07^{s}$ & $01^{h} 39^{m} 07^{s} \pm 0^{s} .3$ & $0^{m} .479$ & $0^{m} .299 \pm 0^{m} .02$ \\
JAN 05 & 2O1P & $00^{h} 19^{m} 26^{s}$ & $00^{h} 22^{m} 01^{s}$ & $0^{m} .136$ & $0^{m} .180 \pm 0^{m} .01$ \\
JAN 09 & 2E3P & $03^{h} 03^{m} 00^{s}$ & $03^{h} 03^{m} 06^{s}$ & $0^{m} .232$ & $0^{m} .253 \pm 0^{m} .01$ \\
JAN 12 & 2O1P & $01^{h} 53^{m} 10^{s}$ & $01^{h} 54^{m} 45^{s}$ & $0^{m} .749$ & $0^{m} .533 \pm 0^{m} .01$ \\
FEB 13 & 2E1A & $02^{h} 04^{m} 44^{s}$ & $02^{h} 05^{m} 21^{s}$ & $0^{m} .712$ & $1^{m} .761 \pm 0^{m} .02$ \\
FEB 23 & 2E1A & $17^{h} 42^{m} 40^{s}$ & $17^{h} 43^{m} 50^{s}$ & $0^{m} .714$ & $0^{m} .628 \pm 0^{m} .01$ \\
MAR 09 & 2E1A & $20^{h} 58^{m} 13^{s}$ & $20^{h} 58^{m} 28^{s}$ & $0^{m} .221$ & $0^{m} .228 \pm 0^{m} .01$ \\
APR 29 & 2E4A & $22^{h} 18^{m} 12^{s}$ & $22^{h} 18^{m} 28^{s}$ & $0^{m} .434$ & $0^{m} .291 \pm 0^{m} .01$ \\
MAY 07 & 3E1P & $20^{h} 29^{m} 11^{s}$ & $20^{h} 29^{m} 06^{s}$ & $0^{m} .446$ & $0^{m} .484 \pm 0^{m} .01$ \\
JUL 04 & 3E1P & $19^{h} 42^{m} 26^{s}$ & $19^{h} 43^{m} 03^{s}$ & $0^{m} .942$ & $1^{m} .351 \pm 0^{m} .01$ \\
\hline
\end{tabular}

Table 5. Relevant data of some mutual events observed during the 1997 campaign (Blanco 1999; Blanco et al. 2001; Arlot et al. 2006). Predicted values of the light minimum (UT) and the light loss of each event are by Arlot 1999.

\begin{tabular}{cccccc}
\hline Date & Event & $\begin{array}{c}\text { Predicted } \\
\text { minimum }\end{array}$ & $\begin{array}{c}\text { Observed } \\
\text { minimum }\end{array}$ & $\begin{array}{c}\text { Predicted } \\
\text { Light loss }(\Delta \mathbf{V})\end{array}$ & $\begin{array}{c}\text { Observed } \\
\text { Light loss }(\Delta \mathbf{V})\end{array}$ \\
\hline JUN 18 & 2E1A & $01^{h} 05^{m} 46^{s}$ & $01^{h} 04^{m} 42^{s} \pm 0^{s} .3$ & $0^{m} .647$ & $1^{m} .25 \pm 0^{m} .01$ \\
JUL 26 & 3O1P & $23^{h} 24^{m} 24^{s}$ & $23^{h} 23^{m} 19^{s}$ & $0^{m} .296$ & $0^{m} .50$ \\
JUL 29 & 1O3P & $23^{h} 50^{m} 43^{s}$ & $23^{h} 49^{m} 30^{s}$ & $0^{m} .206$ & $0^{m} .23$ \\
AUG 01 & 4E3A & $00^{h} 21^{m} 53^{s}$ & $00^{h} 20^{m} 52^{s}$ & $0^{m} .601$ & $0^{m} .95$ \\
AUG 02 & 3O2T & $02^{h} 54^{m} 07^{s}$ & $02^{h} 53^{m} 30^{s}$ & $0^{m} .259$ & $0^{m} .57$ \\
AUG 28 & 1E3P & $00^{h} 37^{m} 25^{s}$ & $00^{h} 37^{m} 25^{s}$ & $0^{m} .199$ & $0^{m} .20$ \\
SEP 29 & 3O2P & $18^{h} 43^{m} 22^{s}$ & $18^{h} 42^{m} 21^{s}$ & $0^{m} .196$ & $1^{m} .22$ \\
OCT 06 & 3O2P & $22^{h} 13^{m} 33^{s}$ & $22^{h} 12^{m} 58^{s}$ & $0^{m} .217$ & $0^{m} .38$ \\
NOV 17 & 3O1P & $16^{h} 48^{m} 30^{s}$ & $16^{h} 47^{m} 35^{s}$ & $0^{m} .278$ & $0^{m} .52$ \\
NOV 18 & 3E1 & $19^{h} 18^{m} 48^{s}$ & $19^{h} 18^{m} 48^{s}$ & $0^{m} .007$ & $0^{m} .01$ \\
\hline
\end{tabular}




\section{PHEMU09}

During the 2009 observational campaign, the 91-cm Cassegrain telescope equipped with the new KODAK KAF 1001E CCD camera (1024x1024 pixels; 24-micron pixelsize) and, for the first time, the 80-cm Cassegrain telescope (APT2) equipped with the KODAK KAF 09000 CCD camera (3056x3056; 12-micron pixel-size) were used. The observations have been carried out in the $B$ band and, depending on the nightly weather conditions and the telescope/CCD set-up used, with exposure time varying from 0.3 to $5 \mathrm{sec}$. Table 6 shows the mutual events observed during the 2009 campaign. Predicted values of the light minimum (UT) and the light loss, for each event, were by http://ftp.imcce.fr/pub/ephem/satel/phemu09/visibility. The acquired data are currently under reduction.

Table 6. Mutual events observed during the 2009 campaign. Predicted values of the light minimum (UT) and the light loss were by http://ftp.imcce.fr/pub/ephem/satel/phemu09/visibility.

\begin{tabular}{cccc}
\hline Date & Event & $\begin{array}{c}\text { Predicted } \\
\text { minimum }\end{array}$ & $\begin{array}{c}\text { Predicted } \\
\text { Light loss }(\Delta \mathbf{V})\end{array}$ \\
\hline JUL 23 & 4E2 & $23^{h} 12^{m} 35^{s}$ & $0^{m} .139$ \\
JUL 24 & 1E2P & $00^{h} 16^{m} 46^{s}$ & $0^{m} .367$ \\
JUL 24 & 1O2P & $01^{h} 15^{m} 09^{s}$ & $0^{m} .214$ \\
SEP 01 & 1O2P & $20^{h} 03^{m} 22^{s}$ & $0^{m} .192$ \\
SEP 01 & 1O2P & $21^{h} 05^{m} 16^{s}$ & $0^{m} .349$ \\
OCT 03 & 1O2P & $18^{h} 31^{m} 24^{s}$ & $0^{m} .161$ \\
OCT 03 & 1E2P & $20^{h} 23^{m} 36^{s}$ & $0^{m} .625$ \\
\hline
\end{tabular}

\section{Acknowledgements}

We are kindly grateful to Dr. G. Bonanno, Dr. G. Leto, P. Bruno, E. Martinetti and, to the technical staff of M. G. Fracastoro Station for their precious assistance during the observations.

\section{References}

Aksnes, K. 1974, Icarus, 21, 100

Aksnes, K. \& Franklin, F. 1984, Icarus, 60, 180

Aksnes, K., Franklin, F., Millis, R., Birch, P., Blanco, C., Catalano, S., \& Piironen, J. 1984, AJ, 89,280

Arlot, J.-E. 1982, A\&A, 107, 305

Arlot, J.-E. 1984, A\&A, 138, 113

Arlot, J.-E., Ruatti, C., Thuillot, W., et al. 1997, A\&SA SS, 125, 399

Arlot, J.-E., Thuillot, W., Ruatti, C., et al. 2006, A\&\&A, 451, 733

Blanco, C. 1988, A\&A, 205, 297

Blanco, C. 1996, Annales de Physique, 21, C1-7

Blanco, C. 1999, Proceeding of the Planetary Science II Italian Meeting, 157, Bormio (IT), January 26-31, 1998, ed. by A. Manara and E. Dotto

Blanco, C. \& Catalano, S. 1974, A\& A, 33, 303

Blanco, C., Riccioli, D., \& Cigna, M. 2001, PESSS, 49, 31

Franklin, F. A., Galilean Satellite Observers, Africano, J. et al. 1991, AJ, 102, 806 